\title{
Serum miR-27a is a biomarker for prognosis of non- small cell lung cancer patients receiving chemotherapy
}

\section{Erfu Xie}

Nanjing Medical University https://orcid.org/0000-0002-6787-7646

Yazhou Ji

Nanjing medical university

Mingxin Lin

Nanjing Medical University

Yuexinzi Jin

Nanjing medical university

Shichang Zhang

Nanjing medical university

Lei Huang

nanjing medical university

Ruihong Sun

Nanjing medical university

Fang Wang

Nanjing medical university

Shiyang Pan ( $\sim$ sypan@njmu.edu.cn )

\section{Research article}

Keywords: NSCLC, miR-27a, prognosis, chemotherapy, individualized treatment

Posted Date: October 23rd, 2020

DOI: https://doi.org/10.21203/rs.2.23892/v2

License: (c) (1) This work is licensed under a Creative Commons Attribution 4.0 International License.

Read Full License

Version of Record: A version of this preprint was published at Translational Cancer Research on January 1st, 2021. See the published version at https://doi.org/10.21037/tcr-20-3276. 


\section{Abstract}

Background: Lung cancer has a high incidence and a 5-year survival rate of less than $15 \%$. Non-small cell lung cancer (NSCLC) accounts for approximately $85 \%$ of lung cancer cases. Chemotherapy and immunotherapy are the most frequently used alternative treatments for patients with advanced-stage NSCLC in whom surgery failed. Previous studies have suggested that miR-27a is involved in cancer development and progression. The purpose of this study was to investigate the clinical value of miR-27a in the prognosis of NSCLC patients after chemotherapy. Methods: Flow cytometry was used to detect the apoptosis rate of SPC-A1 cells treated with optical cisplatin at different times. Simultaneously, the expression of miR-27a in supernatants and cells was detected. Fifty-two newly diagnosed NSCLC patients were recruited. All patients received gemcitabine and cisplatin as first-line chemotherapy and docetaxel as second-line chemotherapy. At the end of every chemotherapy cycle, a therapeutic evaluation was performed according to the RECIST criteria. The expression of serum miR-27a was detected in each cycle. Results: After treatment with $2.5 \mu \mathrm{g} / \mathrm{mL}$ cisplatin, the apoptosis rates of SPC-A1 cells were significantly greater than those of the paired untreated control groups at 12, 24, 48 and $72 \mathrm{~h}$. The expression of miR-27a in supernatants and cells was also consistent with the apoptosis rate and changed a time-dependent manner. The chi-square test showed that an increase in miR-27a after chemotherapy was more common in patients who achieved partial response (PR) than in those who achieved no response (NR) (61.5\% vs $30.8 \%, P=0.026)$. Kaplan-Meier survival analysis indicated that patients with decreased miR-27a levels had poorer outcomes than those with increased miR-27a levels ( $\mathrm{P}$ <0.05). Furthermore, dynamic changes in serum miR-27a with a gradual increasing trend during chemotherapy predicted a good prognosis. Conclusions: Collectively, our results suggest that miR-27a is involved in the apoptosis of lung cancer cells and that serum miR-27a levels are related to the prognosis of NSCLC patients. The expression levels of miR-27a in the serum may be an independent predictor for the prognosis of NSCLC.

\section{Introduction}

Lung cancer is a high incidence respiratory system cancer, and 5-year survival rate is less than $15 \%$. NSCLC is the most common type of lung cancer and it accounts for approximately $85 \%$ of lung cancer cases[1, 2]. Surgery is the most effective treatment for early stage of NSCLC. Whereas, most patients with advanced stage lung cancer failed to receive surgery on account of the low sensitivity and specificity of diagnostic methods[3]. Now, the most alternative treatment for NSCLC are chemotherapy and immunotherapy, which can help to prolong the overall survival of patients and improve the life quality[4]. However, it is necessary to monitor the response of NSCLC patients to immunotherapy and chemotherapy to better improve their prognosis.

MicroRNAs (miRNAs) are 19-25 nucleotide RNAs that have been widely recognized as key mediator in the epigenetic control of gene expression at the post-transcriptional level by base-pairing to the complementary sites on 3'-UTR of the target messenger RNAs (mRNAs) [5]. They play an important regulatory role in a series of biological and pathological processes such as development, differentiation, 
proliferation and apoptosis[6-9]. Similar to the classification of genes, miRNAs can be divided into oncomiRNAs and tumor suppressor miRNAs.

Previously, a monoclonal antibody, which named NJ001, was found by our research. The function of the antibody is specific to NSCLC[10]. In this earlier study, we found that some miRNAs including miR-638 and miR-27a exhibited most significant time dependent expression in response to NJ001 (GEO accession number: GSE51947). Subsequently, we reported that serum miR-638 levels may be considered as an emerging independent predictor for prognosis of NSCLC, because those were related to the survival of NSCLC patients[11]. Moreover, recent publication demonstrated that miR-27a as one of tumor suppressor miRNAs could play a vital role in the progression of cancers and influence tumor cells tumorigenesis, proliferation, apoptosis, invasion, migration and angiogenesis[12-15]. These results showed that cancer development and progression may be associated with miR-27a. Thus, we further worked on discovering the clinical value of miR-27a. Particularly, the purpose of this study is to explore the role of serum miR$27 a$ in predicting the prognosis of NSCLC patients.

\section{Materials And Methods}

Collect cultured supernatants and SPC-A1 cells in vitro experiments

The human lung adenocarcinoma cell line SPC-A1 was purchased from the type culture collection of the Chinese Academy of Sciences, Shanghai, China. Using mass spectrometry by the European Collection of Cell Cultures, SPC-A1 cell line was proven to be uncontaminated by mycoplasma or bacterium. SPC-A1 cells were laid into six-well plates $\left(1.5 \times 10^{6}\right.$ per well). Then, they were treated with complete RPMI 640 medium alone (as the control group) or $2.5 \mathrm{\mu g} / \mathrm{ml}$ of cisplatin and incubated at $37^{\circ} \mathrm{C}$ and $5 \% \mathrm{CO}_{2}$ for 12 , 24,48 or $72 \mathrm{~h}$. The optical treatment concentrations for cisplatin refer to our previous research. Harvesting cultured supernatants and SPC-A1 cells was used for miR-27a detection. The whole experiment was repeated three times, and each time point was set in triplicates.

Detection of apoptosis rates of SPC-A1 cells

Collecting $1 \times 10^{6}$ SPC-A1 cells was detected for apoptosis rate using flow cytometry. According to the manufacturer's instruction, cells were stained with $5 \mu$ propidium iodide and $5 \mu$ l Annexin V-FITC (Annexin V-FITC Apoptosis Detection Kit, BD Biosciences, Franklin, NJ, USA). All analysis was performed on flow cytometry(BD Biosciences).

NSCLC patients and serum specimens

Fifty-two cases of advanced NSCLC patients from March 2010 to May 2012 at the First Affiliated Hospital of Nanjing Medical University were recruited. All patients include 32 males and 20 females, aged 31-77 years (median age: 61 years). All patients with newly diagnosed lung adenocarcinoma were unable to undergo radical surgery and were not treated with radiotherapy or chemotherapy. The diagnosis of lung cancer was confirmed by histopathological examination. All patients received the Gemcitabine and 
Cisplatin as the first-line chemotherapy and Docetaxel as the second-line chemotherapy. At the end of every chemotherapy cycle, a therapeutic evaluation was performed according to the RECIST criteria which including complete response (CR), partial response(PR), stable disease(SD) and progressive disease(PD). SD and PD belong to no response (NR). Patients were followed until March 2013. The present study was approved by the Research Ethics Committee of the First Affiliated Hospital of Nanjing Medical University, and all patients provided written consent prior to study enrollment. With patients' informed consent, Blood was drawn before and after chemotherapy. The whole blood was centrifuged at $2500 \mathrm{~g}$ for $10 \mathrm{~min}$, followed by $16000 \mathrm{~g}$ for $10 \mathrm{~min}$ to completely remove cell debris. Then serum was separated, aliquoted and stored at $-70^{\circ} \mathrm{C}$.

RNA extraction

According to the manufacturer's protocol, total cell supernatants and serum RNA was extracted for Quantitative RT-PCR using the miRNeasy mini kit (QIAgen, Düsseldorf, Germany). Cel-miR-39 as an internal control (59-ucaccggguguaaaucagcuug-39; final concentration: $10^{-5} \mathrm{pmol} / \mathrm{ml}$ ) was added. Cell total RNA was extracted using the QIAzol Lysis Reagent (QIAgen, Düsseldorf, Germany) according to the manufacturer's protocol. A UV spectrophotometer at 260 and $280 \mathrm{~nm}$ and agarose gel electrophoresis were used for validating RNA concentration and quality.

\section{Quantitative RT-PCR}

The miR-27a expression levels of supernatants, cells and serum were detected by quantitative RT-PCR (qRT-PCR). According to the manufacturer's instructions, $5.84 \mu$ reverse transcription (RT) reagents (Applied Biosystems, Foster City, CA) including stem-loop RT primers were mixed with $9.16 \mu$ RNA in each reaction. RT reactions proceeds as follows: first at $16^{\circ} \mathrm{C}$ for $30 \mathrm{~min}$, second at $42^{\circ} \mathrm{C}$ for $30 \mathrm{~min}$, third at $85^{\circ} \mathrm{C}$ for $5 \mathrm{~min}$ and then maintained at $4^{\circ} \mathrm{C}$. Then, in the $\mathrm{ABI} 7500$ real-time PCR system, $10 \mathrm{~min}$ at $95^{\circ} \mathrm{C}$, $15 \mathrm{~s}$ at $95^{\circ} \mathrm{C}$ with $45 \mathrm{cycles}$ and $1 \mathrm{~min}$ at $60^{\circ} \mathrm{C}$. The expression level of miR-27a in serum was expressed as the multiple change of normalized expression of cel-miR-39. The formula was $2^{-\Delta \mathrm{Ct}}$ in which $\triangle \mathrm{Ct}=$ $\mathrm{Ct}_{\mathrm{miR}-27 \mathrm{a}}-\mathrm{Ct}_{\mathrm{miR}-39}$. In the supernatants, the expression level of miR-27a was expressed as the multiple change of normalized cel-miR-39 expression. The formula was $2^{-} \Delta \triangle \mathrm{Ct}$ in which $\triangle \triangle \mathrm{Ct}=\left(\mathrm{Ct}_{\mathrm{miR}-27 a}{ }^{-} \mathrm{Ct}_{\mathrm{miR}-}\right.$

$\left.{ }_{39}\right)_{\text {treated }}-\left(\mathrm{Ct}_{\mathrm{miR}-27 a}-\mathrm{Ct}_{\mathrm{miR}-39}\right)_{\text {untreated. }}$ The expression level of miR-27a in cells was expressed by the formula in multiple changes from normal to u6snRNA expression. The formula was $2^{-} \triangle \triangle \mathrm{Ct}$ in which $\triangle \triangle \mathrm{Ct}=$ $\left(\mathrm{Ct}_{\text {miR-27a }}-\mathrm{Ct}_{\mathrm{U6}}\right)_{\text {treated }}-\left(\mathrm{Ct}_{\mathrm{miR}-27 \mathrm{a}}-\mathrm{Ct}_{\mathrm{U6}}\right)_{\text {untreated }}$.

Statistical analysis

SPSS 16.0 software (Chicago, IL, USA) and GraphPad Prism 7.0 (San Diego, CA, USA) were used for statistical analyses. Proportions of miR-27a expression levels change before and after chemotherapy between PR and NR (including PD and SD) populations were compared using the chi-square test. The Kaplan-Meier method was used for survival analysis. We compare survival times between groups by the log-rank test. A $P$-value $<0.05$ was considered statistically significant. 


\section{Results}

Apoptosis of SPC-A1 cells induced by Cisplatin.

SPC-A1 cells were treated with $2.5 \mu \mathrm{g} / \mathrm{ml}$ cisplatin for different periods of time for evaluating the effect of cisplatin on SPC-A1 apoptosis. Flow cytometry was used to detect the apoptosis rate at $12 \mathrm{~h}, 24 \mathrm{~h}, 48 \mathrm{~h}$ and $72 \mathrm{~h}$ after treatment with or without cisplatin. Comparing with those of paired untreated control groups, the morphology of SPC-A1 cells were changed (Fig. 1). Moreover, the apoptosis rates of the SPCA1 cells cultured for 12, 24, 48 and $72 \mathrm{~h}$ with cisplatin were significantly greater (Figs. 2 and 3 ). With prolonged exposure with cisplatin, these apoptosis rates increased more markedly. It is suggested that cisplatin can induce apoptosis of SPC-A1 cells in a time-dependent manner.

MiR-27a expression increased in SPC-A1 cells cultured supernatants in a time-dependent manner with cisplatin treatment.

MiR-27a expression levels in the culture supernatants of SPC-A1 cells treated with cisplatin were detected by qRT-PCR. We revealed that the miR-27a levels in the SPC-A1 cultured supernatants after being treated with cisplatin a concentration of $2.5 \mu \mathrm{g} / \mathrm{mL}$ for $72 \mathrm{~h}$ were more four-fold higher than those in the untreated control group (Fig. 4a). MiR-27a expression levels in cisplatin ( $2.5 \mu \mathrm{g} / \mathrm{mL})$ group increased gradually at $12 \mathrm{~h}, 24 \mathrm{~h}, 48 \mathrm{~h}$ and $72 \mathrm{~h}$, and the difference between any time period of two groups was significant $(P<0.05)$. When $2.5 \mu \mathrm{g} / \mathrm{mL}$ cisplatin was used for treatment for different periods of time $(12-$ $72 \mathrm{~h}$ ), the levels of miR-27a in supernatants increased with time dependence (Fig. 4a).

MiR-27a expression increased in SPC-A1 cells in a time-dependent manner with cisplatin treatment.

MiR-27a expression levels in SPC-A1 cells treated with cisplatin were detected by qRT-PCR. The results showed that the expression level of miR-27a in SPC-A1 cells treated with $2.5 \mu \mathrm{g} / \mathrm{mL}$ cisplatin for 72 hours was more 8 times higher than that in untreated control cells(Fig. 4b). Cells miR-27a expression levels in cisplatin $(2.5 \mu \mathrm{g} / \mathrm{mL})$ group increased between $12 \mathrm{~h}$ and $24 \mathrm{~h}$, and miR-27a expression levels between the two group were significantly different $(P<0.05)$. However, there was no significant change in $24 \mathrm{~h}, 48 \mathrm{~h}$ and $72 \mathrm{~h}$. This result showed that cells miR-27a expression levels in cisplatin $(2.5 \mu \mathrm{g} / \mathrm{mL})$ group entered into the platform after $24 \mathrm{~h}$ (Fig. 4b).

Correlation between prognosis and the change of miR-27a expression levels before and after chemotherapy in NSCLC patients.

As it demonstrated that, accompanying the apoptosis of SPC-A1 cells, miR-27a expression levels increased in SPC-A1 culture supernatants with time dependence after cisplatin treatment. Therefore, it is necessary to assess the prognostic value of in NSCLC patients who receiving first-line chemotherapy. MiR-27a expression levels of 52 patients receiving first-line chemotherapy in serum were monitored before and after chemotherapy. The results showed that the changes include 24 patients increasing and 28 patients decreasing. The follow-up completed until March 2013. The outcome of the patients includes 26 patients PR, 26 patients NR (include 10 patients SD and 16 patients PD). The detail information is 
shown in Table 1. The chi-square test showed significantly elevation of miR-27a after chemotherapy was more common in patients with PR compared with the patients who achieved NR $(61.5 \%$ vs $30.8 \%, P=$ 0.026). This result found that there was a certain correlation between serum miR-27a levels increasing after chemotherapy and the better outcome of NSCLC patient.

\section{Table 1}

Correlation between prognosis and the change of miR-27a expression levels before and after chemotherapy in lung adenocarcinoma patients $(n=52)$.

\begin{tabular}{|llll|}
\hline Prognosis & \multicolumn{2}{l}{ miR-27a change after chemotherapy } & P Value \\
\cline { 2 - 3 } & \multicolumn{1}{l}{ Increasing } & Decreasing & \multirow{2}{*}{0.026} \\
\hline PR & 16 & 10 & \\
\hline NR(SD + PD) & 8 & 18 & \\
\hline PR: partial response,NR: no response,SD:stable disease, PD: progressive disease. & \\
\hline
\end{tabular}

Correlation between miR-27a levels and NSCLC patients' survival outcome.

At the end of follow-up, 52 patients who completed first-line chemotherapy were divided into two groups according to the increase or decrease of serum miR-27a after chemotherapy. The patients increased by 24 cases after chemotherapy and decreased by 28 cases. Kaplan-Meier survival analysis showed that after first-line chemotherapy, the average survival time of 24 patients with increasing serum miR-27a was 28.4 months, while the average survival time was 9 months for the decreasing 28 patients; The survival time in increasing group is significantly higher than the decreasing group, and the difference between the two is significant $(P=0.0051)$. As showed in Fig. 5 , the result suggested that miR-27a decrease patients had poor outcome compared with the increase patients.

Dynamic changes in serum miR-27a levels during chemotherapy.

The dynamic changes of serum mir-27a in 4 patients with NSCLC after different chemotherapy cycles were monitored. As showed in Fig. 6, patient A received 3 cycles of first-line drug therapy. Computed tomography (CT) showed that the tumor was significantly reduced, while tumor markers decreased and serum miR-27a levels increased gradually. At the end of follow-up, the patient survived and showed PR. Patient $B$ received 3 cycles of first-line chemotherapy, but due to the disease progression of multiple metastases throughout the body, 10 months later, the relative content of serum miR-27a continued to decrease during chemotherapy. After patient $\mathrm{C}$ ended the first-line drug chemotherapy for 1 cycle, the progress of the disease was changed to second-line drug chemotherapy. After 3 cycles of second-line chemotherapy, the relative content of serum miR-27a continued to increase. By the end of follow-up, the patient's conditions show PR. Patient D received 2 cycles of first-line drug chemotherapy, the condition was stable, and was allowed to be discharged. After 1 month, the disease progressed and the relative content of serum miR-27a was significantly decreased. Chemotherapy was used instead of second-line chemotherapy, and the patient died and survived for 11 months. The above 4 patients also demonstrated 
that miR-27a can be used as a tumor marker to guide individualized treatment efficacy during chemotherapy.

\section{Discussion}

MicroRNAs have been discovered in decades, which can play a role in negatively regulating gene expression by recognizing the $3^{\prime} U T R$ of a specific target mRNA and inhibiting the translation at the posttranscriptional level [16]. Recent Studies have revealed that miRNAs have impact on the regulation of tumor biological behavior in lung cancer and miRNAs detection has been widely used in early diagnosis [17], prognosis [18] and therapeutic evaluation [19], as well as chemosensitivity [20] or chemoresistance[21].

Recently, miRNAs have been widely studies as an important means of epigenetic regulation [22]. A considerable amount of studies have showed that the dysregulation of miRNAs in NSCLC may involve in development and progression of NSCLC Therefore, miRNAs could serve as promising molecular markers for clinical application [23]. For example, Pan $\mathrm{J}$ et al revealed that miR-33a-5p and miR-128-3p were more sensitive/specific than traditional tumor markers such as NSE, CYFRA21-1 and CA72-4 and could serve as novel biomarkers for the early detection of lung cancer[24]. Yang $M$ et al indicated that miR-21 and miR-155 show promising associations with prognosis in lung cancer and could predict recurrence and poor survival in NSCLC[25]. MiR-27 family is composed of miR-27a and miR-27b, and miR-27a plays an important role in tumor development. In recent studies, miR-27a, was significantly dysregulated in various cancers, such as liver cancer [26], NSCLC [27] and prostate cancer [28], and acted as an oncogene. Meanwhile, it could function as a tumor suppressor in bladder cancer [29], gastric cancer [30], esophageal squamous-cell [31]. These contradictory studies have also prompted us to reveal the role of miR-27a in tumors and its potential clinical applications.

Using a previous miRNAs expression microarray analysis, we found that miR-27a expression levels in SPC-A1 cells treated with NJ001 increased with time dependence. To further discover clinical application of miR-27a in NSCLC, the apoptosis rates of SPC-A1 cells treated with $2.5 \mu \mathrm{g} / \mathrm{mL}$ cisplatin at different periods of time in vitro were measured. Simultaneously, miR-27a expression levels in culture supernatants and cells were measured. Our results suggested that SPC-A1 apoptosis were induced by cisplatin with time dependence which is similar to the research of Shi L et al[32]. Importantly, the apoptosis rates were positively associated with a increase in miR-27a expression with time dependence both in the SPC-A1 cells and in culture supernatants. The expression of miR-27a increased with the increase of exposure time and apoptosis rates, which suggests that mir-27a may play an active role in apoptosis.

The serum miR-27a levels of 52 lung adenocarcinoma patients were also measured before and after chemotherapy by qRT-PCR analysis. we found that the proportion of serum miR-27a levels increasing was $61.5 \%$ in PR patients group compared with $30.8 \%$ in NR patient group after the first chemotherapy cycle. This result suggested that there was a correlation between serum miR-27a levels increasing after chemotherapy and the outcome of NSCLC patient. Furthermore, Kaplan-Meier survival analysis showed 
that after first-line chemotherapy, the average survival time of patients with increasing and decreasing of serum miR-27a were 28.4 months vs 9 months. The result suggested that miR-27a decreasing patients have poor outcome compared with the increasing patients.

Previous research showed that the dynamic changes of miRNAs are potentiality used to discriminate types of response to antitumor therapy in lung cancer patients [33] and CTC counts can be used for the dynamic monitoring and prediction of outcome in EGFR-mutated NSCLC patients treated with EGFRTKIs[34]. Inspired by these researches, we evaluated the dynamic changes of the expression of miR-27a in the serum of the representative patients before and after chemotherapy. The results showed that the level of serum miR-27a in PR group increased gradually (patient A and patient C), while that in SD group and PD group decreased(patient B). Hence, the results revealed that the dynamic changes of miR-27a level in these individualized cases combined with the therapeutic evaluation can be used to predict the outcome of patients.

Liquid biopsy included circulating tumor cells (CTCs), circulating free DNA (cfDNA), miRNAs, exosomes and tumor-educated platelets (TEP) was potential use for non-invasive screening, early diagnosis, prognosis, response to treatment and real time monitoring of the disease, in NSCLC patients [35]. Recently, many studies have demonstrated that a considerable amount of miRNAs could be prognostic tools[36, 37]. MiR-27a is a member of the well-identified miRNAs in tumors, and its diverse function is mainly depended on the cancer types, which could play a role either in promoting cancer or in tumor suppression [38]. In our study, there is a positive correlation between the expression of miR-27a and apoptosis of tumor cells. Moreover, patients with miR-27a increasing after the first cycle chemotherapy have better outcome than patients with miR-27a decreasing. These have confirmed that miR-27a may play a role in tumor suppression in lung cancer progress, which similar to result of Acunzo $\mathrm{M}$ et al [27], but contradict to the research of Chae DK et al [39]. These contradiction research need to be confirmed by further research in future.

In conclusion, we found that the expression of miR-27a in serum of NSCLC patients changed after the first chemotherapy, which was related to the prognosis of NSCLC. More precisely, the increase of serum miR-27a is related to the better prognosis of NSCLC patients. Dynamic changes of serum miR-27a after for each cycle of chemotherapy showing gradual increase predict the patients with a better clinical outcome.

Serum miR-27a may be a potential prognostic biomarker for NSCLC patients. However, the potential molecular mechanism of miR-27a in lung cancer and its therapeutic target for NSCLC need further study.

\section{Declarations}

\section{Acknowledgments}

This study was supported by grants from Key Laboratory for Laboratory Medicine of Jiangsu Province of China (No. ZDXKB2016005), the National Natural Science Foundation of China $(81101322,81672100)$. 
Conflicts of Interest

The authors have no conflicts of interest to declare.

\section{References}

1. Miller KD, Nogueira L, Mariotto AB, Rowland JH, Yabroff KR, Alfano CM, Jemal A, Kramer JL, Siegel RL: Cancer treatment and survivorship statistics, 2019. CA: a cancer journal for clinicians 2019, 69(5):363-385.

2. Siegel RL, Miller KD, Jemal A: Cancer statistics, 2019. CA: a cancer journal for clinicians 2019, 69(1).

3. Altorki NK, Kamel MK, Narula N, Ghaly G, Nasar A, Rahouma M, Lee PC, Port JL, Stiles BM: Anatomical Segmentectomy and Wedge Resections Are Associated with Comparable Outcomes for Patients with Small cT1N0 Non-Small Cell Lung Cancer. Journal of thoracic oncology : official publication of the International Association for the Study of Lung Cancer 2016, 11(11):1984-1992.

4. Tabchi S, Kassouf E, Rassy EE, Kourie HR, Martin J, Campeau M-P, Tehfe M, Blais N: Management of stage III non-small cell lung cancer. Seminars in oncology 2017, 44(3):163-177.

5. Zhang J, Raju GS, Chang DW, Lin SH, Chen Z, Wu X: Global and targeted circulating microRNA profiling of colorectal adenoma and colorectal cancer. Cancer 2018, 124(4):785-796.

6. Bhaskaran M, Mohan M: MicroRNAs: history, biogenesis, and their evolving role in animal development and disease. Veterinary pathology 2014, 51(4):759-774.

7. Wang J, Liu S, Li J, Zhao S, Yi Z: Roles for miRNAs in osteogenic differentiation of bone marrow mesenchymal stem cells. Stem cell research \& therapy 2019, 10(1):197.

8. Wang M, Liu C, Su Y, Zhang K, Zhang Y, Chen M, Ge M, Gu L, Lu T, Li N et al: miRNA-34c inhibits myoblasts proliferation by targeting YY1. Cell cycle (Georgetown, Tex) 2017, 16(18):1661-1672.

9. Song Y-S, Joo H-W, Park I-H, Shen G-Y, Lee Y, Shin JH, Kim H, Kim K-S: Bone marrow mesenchymal stem cell-derived vascular endothelial growth factor attenuates cardiac apoptosis via regulation of cardiac miRNA-23a and miRNA-92a in a rat model of myocardial infarction. PloS one 2017, 12(6):e0179972.

10. Pan S, Wang F, Huang P, Xu T, Zhang L, Xu J, Li Q, Xia W, Sun R, Huang L et al: The study on newly developed McAb NJ001 specific to non-small cell lung cancer and its biological characteristics. PloS one 2012, 7(3):e33009.

11. Wang F, Lou J-f, Cao Y, Shi X-h, Wang P, Xu J, Xie E-f, Xu T, Sun R-h, Rao J-y et al: miR-638 is a new biomarker for outcome prediction of non-small cell lung cancer patients receiving chemotherapy. Experimental \& molecular medicine 2015, 47:e162.

12. Li X, Xu M, Ding L, Tang J: MiR-27a: A Novel Biomarker and Potential Therapeutic Target in Tumors. Journal of Cancer 2019, 10(12):2836-2848. 
13. Li Y, Li J, Sun X, Chen J, Sun X, Zheng J, Chen R: MicroRNA-27a functions as a tumor suppressor in renal cell carcinoma by targeting epidermal growth factor receptor. Oncology letters 2016, 11(6):4217-4223.

14. Bao Y, Chen Z, Guo Y, Feng Y, Li Z, Han W, Wang J, Zhao W, Jiao Y, Li K et al: Tumor suppressor microRNA-27a in colorectal carcinogenesis and progression by targeting SGPP1 and Smad2. PloS one 2014, 9(8):e105991.

15. Wang YL, Gong WG, Yuan QL: Effects of miR-27a upregulation on thyroid cancer cells migration, invasion, and angiogenesis. Genetics and molecular research : GMR 2016, 15(4).

16. Bartel DP: MicroRNAs: genomics, biogenesis, mechanism, and function. Cel/2004, 116(2):281-297.

17. Zhang Y, Sui J, Shen X, Li C, Yao W, Hong W, Peng H, Pu Y, Yin L, Liang G: Differential expression profiles of microRNAs as potential biomarkers for the early diagnosis of lung cancer. Oncology reports 2017, 37(6):3543-3553.

18. Qi L, Gao C, Feng F, Zhang T, Yao Y, Wang X, Liu C, Li J, Li J, Sun C: MicroRNAs associated with lung squamous cell carcinoma: New prognostic biomarkers and therapeutic targets. Journal of cellular biochemistry 2019.

19. Yuwen DL, Sheng BB, Liu J, Wenyu W, Shu YQ: MiR-146a-5p level in serum exosomes predicts therapeutic effect of cisplatin in non-small cell lung cancer. European review for medical and pharmacological sciences 2017, 21(11):2650-2658.

20. Deng $H$, Qianqian G, Ting J, Aimin Y: miR-539 enhances chemosensitivity to cisplatin in non-small cell lung cancer by targeting DCLK1. Biomedicine \& pharmacotherapy = Biomedecine \& pharmacotherapie 2018, 106:1072-1081.

21. Filipska M, Skrzypski M, Czetyrbok K, Stokowy T, Stasiłojć G, Supernat A, Jassem J, Żaczek AJ, Bigda $\mathrm{J}:$ MiR-192 and miR-662 enhance chemoresistance and invasiveness of squamous cell lung carcinoma. Lung cancer (Amsterdam, Netherlands) 2018, 118:111-118.

22. Mehta A, Dobersch S, Romero-Olmedo AJ, Barreto G: Epigenetics in lung cancer diagnosis and therapy. Cancer metastasis reviews 2015, 34(2):229-241.

23. Hashemi ZS, Khalili S, Forouzandeh Moghadam M, Sadroddiny E: Lung cancer and miRNAs: a possible remedy for anti-metastatic, therapeutic and diagnostic applications. Expert review of respiratory medicine 2017, 11(2):147-157.

24. Pan J, Zhou C, Zhao X, He J, Tian H, Shen W, Han Y, Chen J, Fang S, Meng X et al: A two-miRNA signature (miR-33a-5p and miR-128-3p) in whole blood as potential biomarker for early diagnosis of lung cancer. Scientific reports 2018, 8(1):16699.

25. Yang M, Shen H, Qiu C, Ni Y, Wang L, Dong W, Liao Y, Du J: High expression of miR-21 and miR-155 predicts recurrence and unfavourable survival in non-small cell lung cancer. European journal of cancer (Oxford, England : 1990) 2013, 49(3):604-615.

26. Li S, Li J, Fei BY, Shao D, Pan Y, Mo ZH, Sun BZ, Zhang D, Zheng X, Zhang M et al: MiR-27a promotes hepatocellular carcinoma cell proliferation through suppression of its target gene peroxisome proliferator-activated receptor Y. Chinese medical journal 2015, 128(7):941-947. 
27. Acunzo M, Romano G, Palmieri D, Laganá A, Garofalo M, Balatti V, Drusco A, Chiariello M, NanaSinkam $P$, Croce CM: Cross-talk between MET and EGFR in non-small cell lung cancer involves miR27a and Sprouty2. Proceedings of the National Academy of Sciences of the United States of America 2013, 110(21):8573-8578.

28. Fletcher CE, Dart DA, Sita-Lumsden A, Cheng H, Rennie PS, Bevan CL: Androgen-regulated processing of the oncomir miR-27a, which targets Prohibitin in prostate cancer. Human molecular genetics 2012, 21(14):3112-3127.

29. Drayton RM, Dudziec E, Peter S, Bertz S, Hartmann A, Bryant HE, Catto JW: Reduced expression of miRNA-27a modulates cisplatin resistance in bladder cancer by targeting the cystine/glutamate exchanger SLC7A11. Clin Cancer Res 2014, 20(7):1990-2000.

30. Zhao $X$, Yang L, Hu J: Down-regulation of miR-27a might inhibit proliferation and drug resistance of gastric cancer cells. Journal of experimental \& clinical cancer research : CR 2011, 30:55.

31. Wu XZ, Wang KP, Song HJ, Xia JH, Jiang Y, Wang YL: MiR-27a-3p promotes esophageal cancer cell proliferation via F-box and WD repeat domain-containing 7 (FBXW7) suppression. International journal of clinical and experimental medicine 2015, 8(9):15556-15562.

32. Shi L, Xu Z, Wu G, Chen X, Huang Y, Wang Y, Jiang W, Ke B: Up-regulation of miR-146a increases the sensitivity of non-small cell lung cancer to DDP by downregulating cyclin J. BMC cancer 2017, 17(1):138.

33. Ponomaryova AA, Morozkin ES, Rykova EY, Zaporozhchenko IA, Skvortsova TE, Dobrodeev A Y, Zavyalov AA, Tuzikov SA, Vlassov VV, Cherdyntseva NV et al: Dynamic changes in circulating miRNA levels in response to antitumor therapy of lung cancer. Experimental lung research 2016, 42(2):95102.

34. Jiang T, Zhao J, Zhao C, Li X, Shen J, Zhou J, Ren S, Su C, Zhou C, O'Brien M: Dynamic Monitoring and Predictive Value of Circulating Tumor Cells in EGFR-Mutated Advanced Non-Small-Cell Lung Cancer Patients Treated With First-Line EGFR Tyrosine Kinase Inhibitors. Clinical lung cancer 2019, 20(2):124-133.e122.

35. Pérez-Callejo D, Romero A, Provencio M, Torrente M: Liquid biopsy based biomarkers in non-small cell lung cancer for diagnosis and treatment monitoring. Translational lung cancer research 2016, 5(5):455-465.

36. Nagaraj $A B$, Joseph P, DiFeo A: miRNAs as prognostic and therapeutic tools in epithelial ovarian cancer. Biomarkers in medicine 2015, 9(3):241-257.

37. Yu C, Wan H, Shan R, Wen W, Li J, Luo D, Wan R: The Prognostic Value of the MiR-200 Family in Colorectal Cancer: A Meta-analysis with 1882 Patients. Journal of Cancer 2019, 10(17):4009-4016.

38. Tian Y, Fu S, Qiu GB, Xu ZM, Liu N, Zhang XW, Chen S, Wang Y, Sun KL, Fu WN: MicroRNA-27a promotes proliferation and suppresses apoptosis by targeting PLK2 in laryngeal carcinoma. BMC cancer 2014, 14:678.

39. Chae DK, Ban E, Yoo YS, Kim EE, Baik JH, Song EJ: MIR-27a regulates the TGF- $\beta$ signaling pathway by targeting SMAD2 and SMAD4 in lung cancer. Molecular carcinogenesis 2017, 56(8):1992-1998. 


\section{Figures}
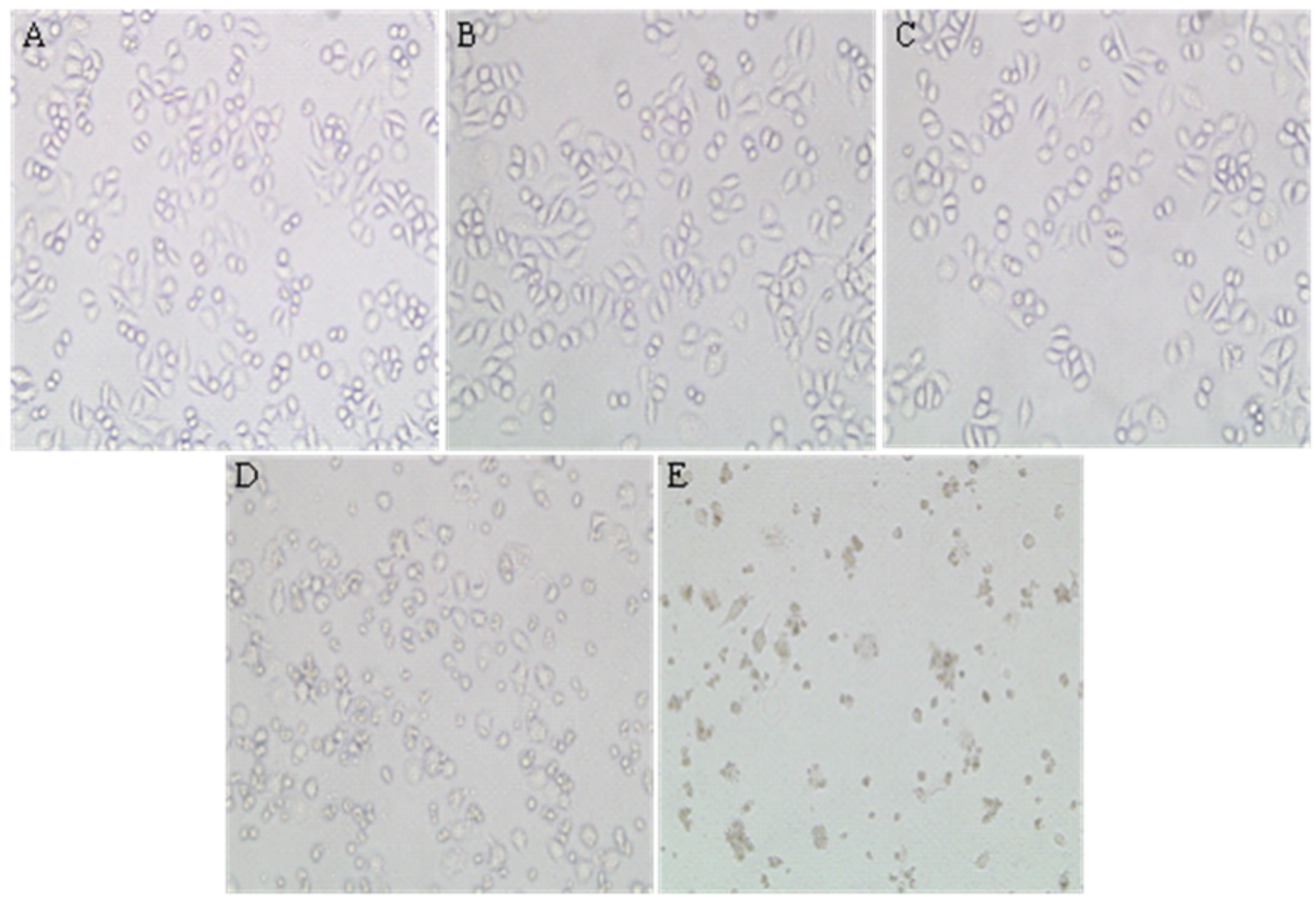

\section{Figure 1}

The morphology changes of SPC-A1 cells treated with $2.5 \mu \mathrm{g} / \mathrm{mL}$ cisplatin $(\times 200)$. A: Blank control; B: $12 \mathrm{~h}$; C: 24h; D: 48h; E: 72h. 


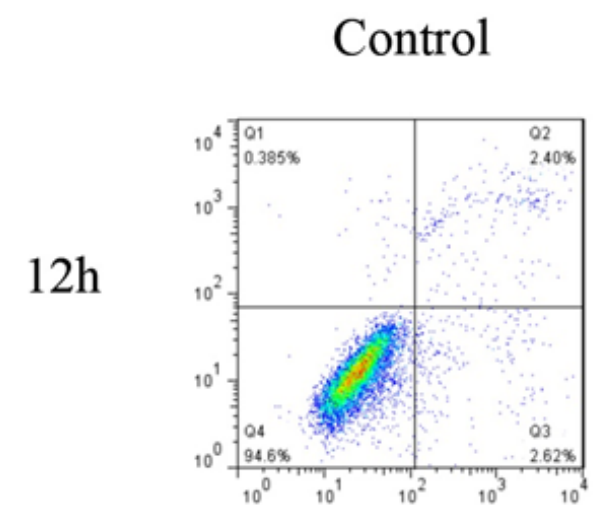

\section{$2.5 \mu \mathrm{g} / \mathrm{mL}$}
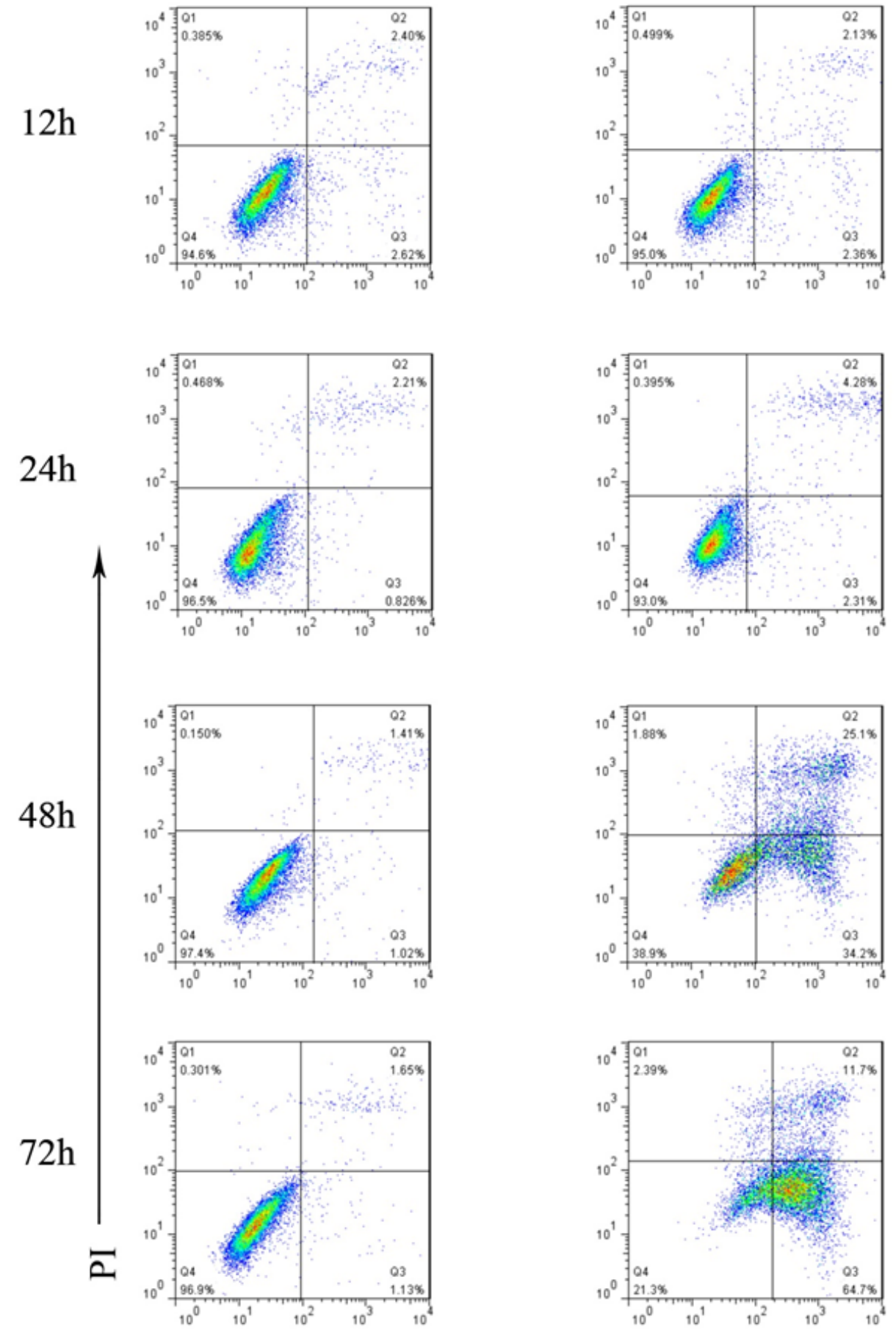

Annexin V-FITC

\section{Figure 2}

The original flow cytometry plot of SPCA1 cells at different time points post treatment with $2.5 \mu \mathrm{g} / \mathrm{mL}$ cisplatin. 


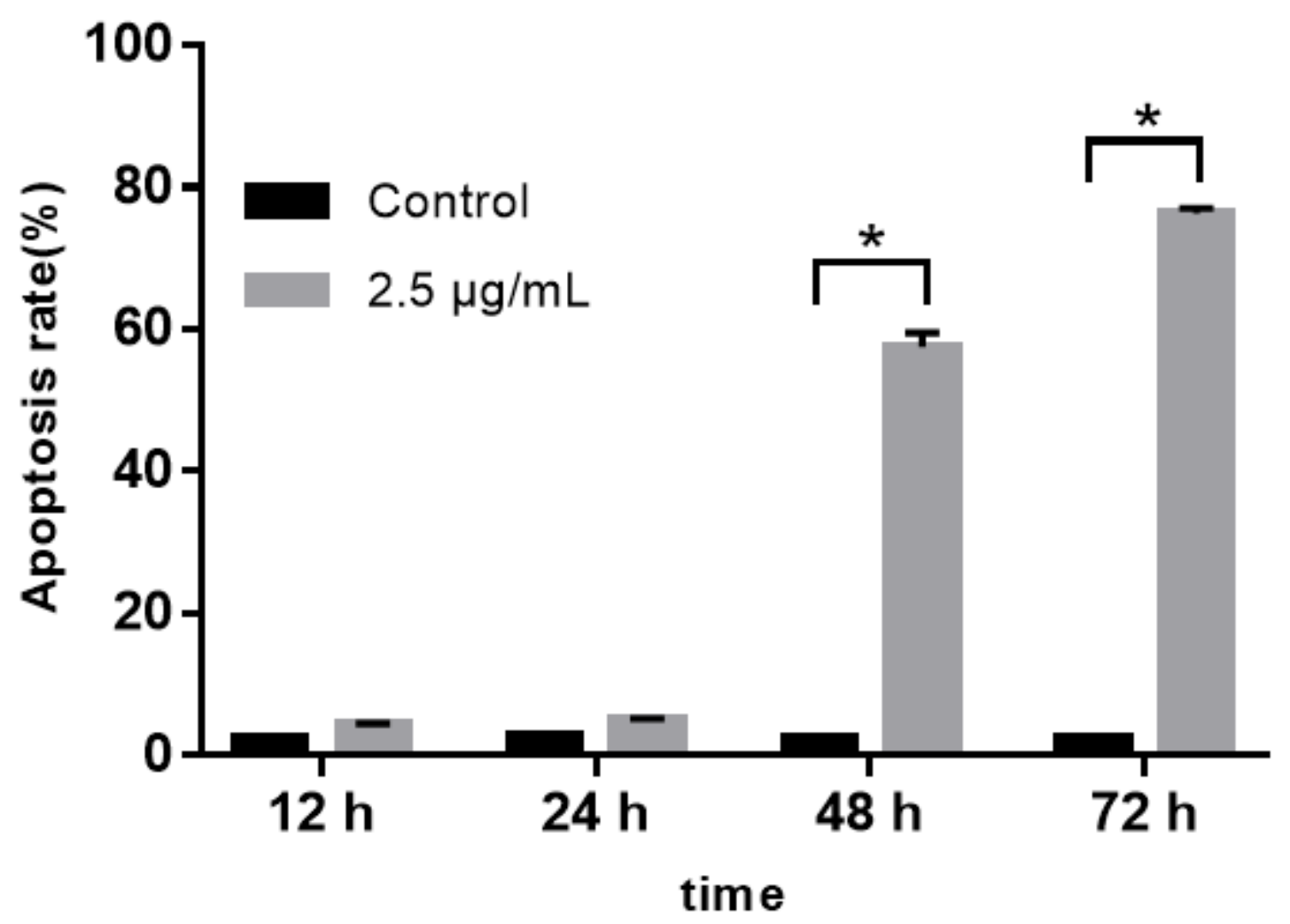

Figure 3

Apoptosis rates of SPC-A1 cells at different time points post treatment with $2.5 \mu \mathrm{g} / \mathrm{mL}$ cisplatin. $\left({ }^{\star} \mathrm{P}<0.05\right)$ 

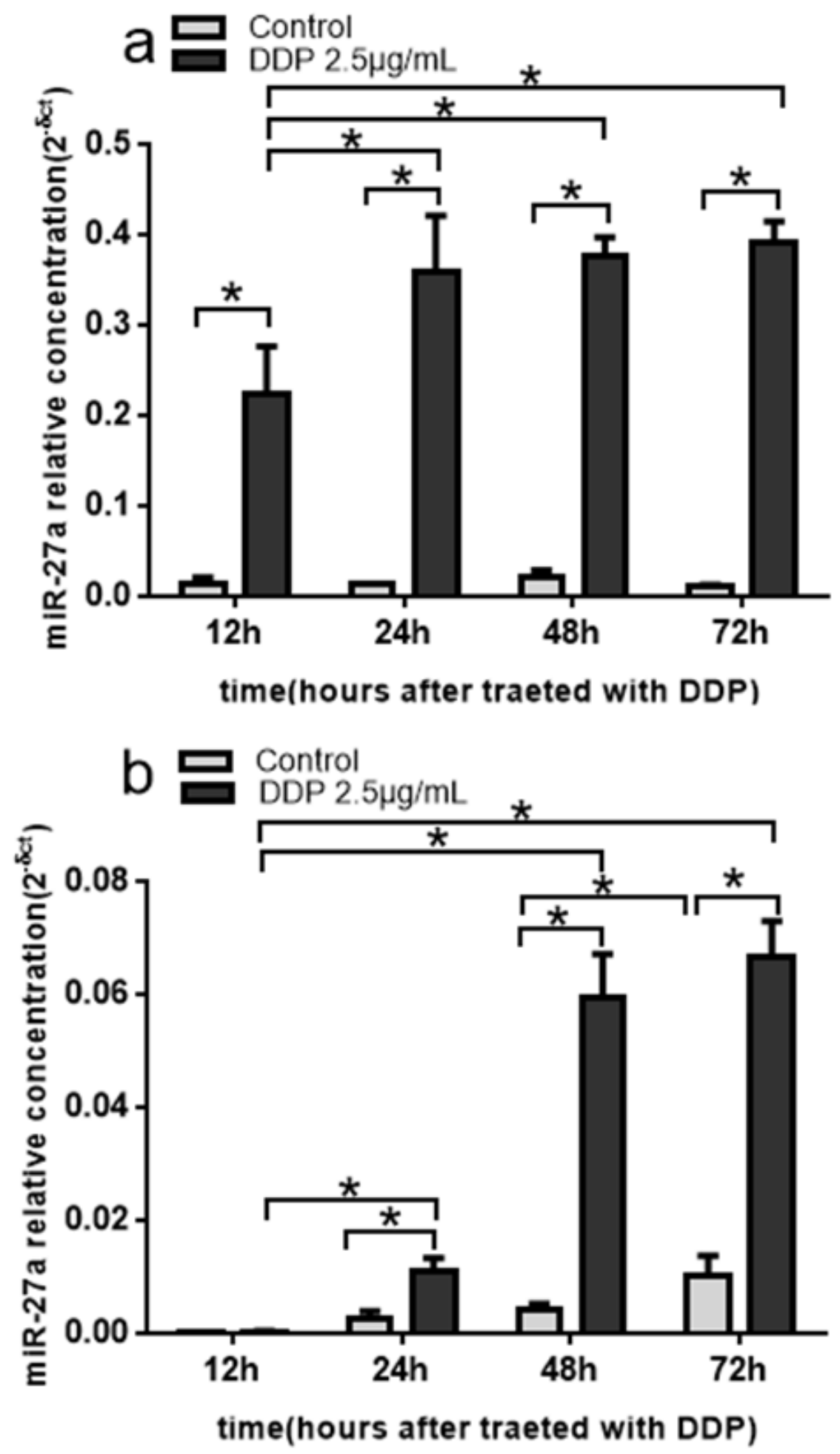

Figure 4

Relative levels of miR-27a expression in SPC-A1 cultured supernatants and cells treated with cisplatin of $2.5 \mu \mathrm{g} / \mathrm{mL}$. a.cultured supernatants; b. SPC-A1 cells. 


\section{Two groups}

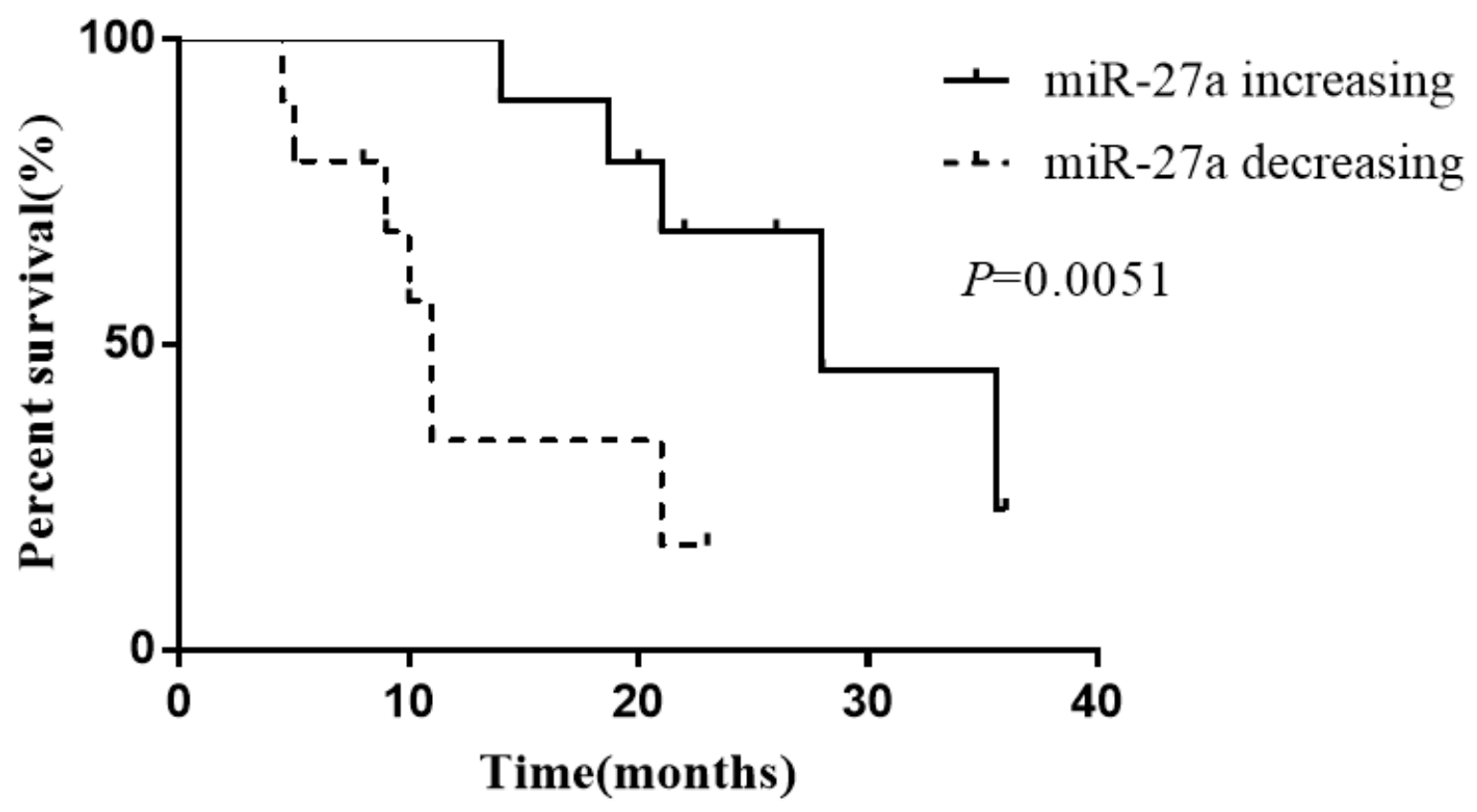

Figure 5

Kaplan-Meier survival curve for overall survival of NSCLC patients $(n=52)$. Kaplan-Meier analysis was performed to evaluate the overall survival of NSCLC patients according to miR-27a expression patterns after one cycle of first-line chemotherapy, in which 24 patients had increasing miR-27a expression levels, and 28 patients had decreasing miR-27a expression levels. 

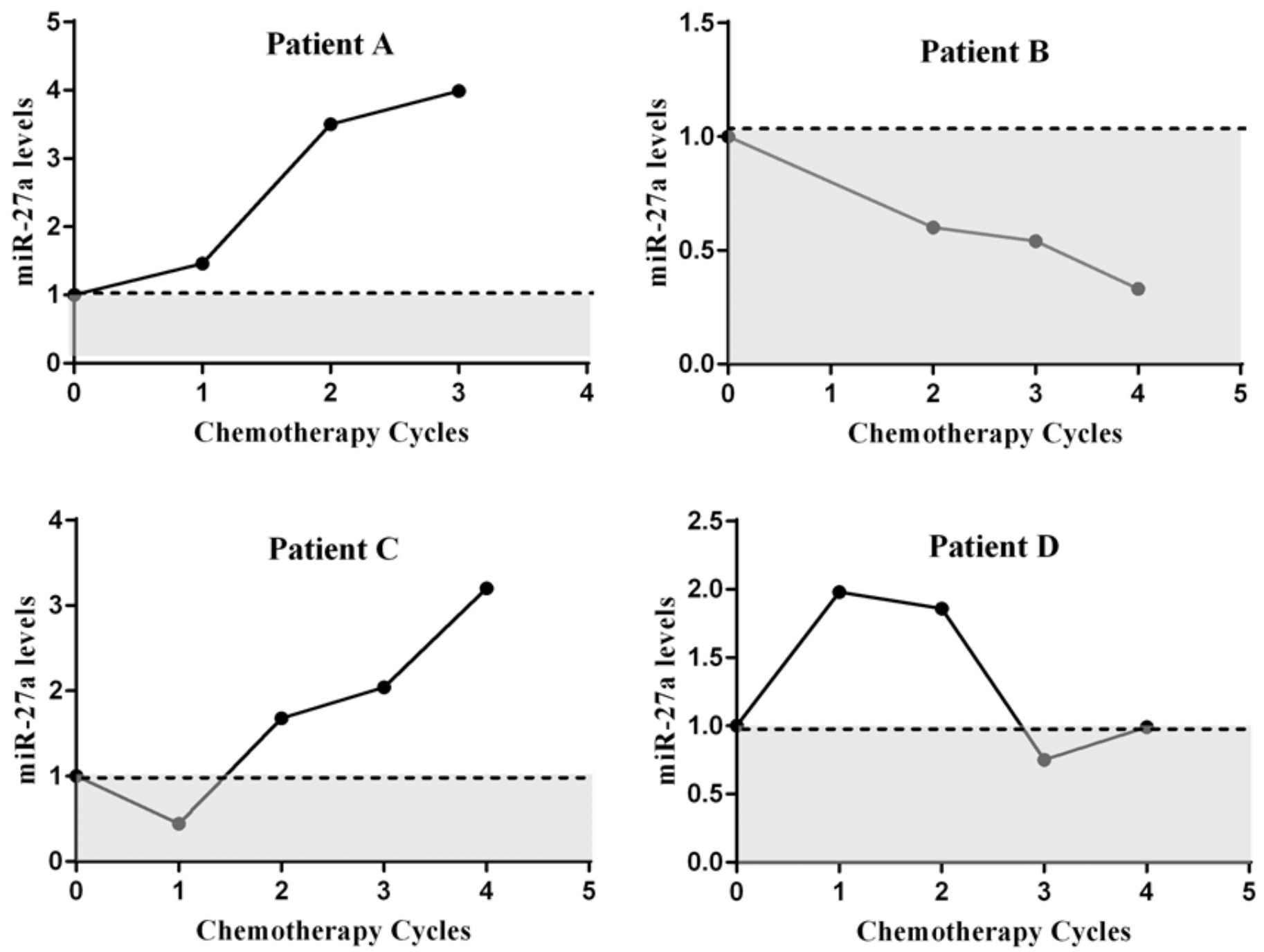

Figure 6

Dynamic changes of miR-27a levels at different time points following chemotherapy. 